\title{
Down-regulation of AKT combined with radiation-induced autophagy and apoptosis roles in MCF-7 cells ${ }^{1}$
}

\author{
Jing Zhang ${ }^{\mathrm{a}}$, Dali Zhao ${ }^{\mathrm{b}}$, Zhongwei Xie ${ }^{\mathrm{b}}$ and Yali Qi ${ }^{\mathrm{a}}{ }^{\text {** }}$ \\ ${ }^{a}$ Department of Epidemiology, School of Public Health, Beihua University, Jilin 132011, Jilin, China \\ ${ }^{b}$ Jilin Entry- exit Inspection and Quarantine, Jilin 132011, Jilin, China
}

\begin{abstract}
Autophagy is an evolutionarily conservation process whereby cytoplasm and cellular organelles are degraded in lysosomes for amino acid and energy recycling. Autophagy concurrent with radiotherapy has emerged as a novel approach in breast cancer treatment. Our studies conclude that autophagy and apoptosis can be induced by radiation and inhibition of autophagy can increase apoptosis. In addition, Akt is a molecule that down-regulates autophagy and apoptosis; blocking Akt can enhance autophagy and apoptosis induced by radiation in MCF-7 cells. Akt could become a new focus in breast cancer radiotherapy.
\end{abstract}

Keywords: Autophagy, apoptosis, radiation, Akt, breast cancer

\section{Introduction}

Breast cancer is the most common cancer among females and one of the leading causes of death, which occupies $30 \%$ of new women malignancies [1]. Radiotherapy is a well-developed targeting technology for cancer; it kills tumor cell by necrosis and apoptosis [2]. Radiotherapy for breast cancer can directly induce apoptosis and cell cycle arrest, which has extraordinary significance for breast cancer treatment [3]. Radiotherapy efficacy is limited by tumor radiation resistance, inducing apoptosis through ionizing radiation is less effective when cancer cells lack apoptosis gene caspase-3 and -9 , such as MCF-7 cell lines $[4,5]$.

Autophagy is another type of programmed cell death (PCD). It is an evolutionarily conservation intracellular catabolic process in which a cell degrades long-lived proteins and damaged organelles [6]. It can be triggered by a wide spectrum of cellular stress, including nutrient deprivation, genotoxins, hypoxia, ionizing radiation (IR), and chemotherapeutic drugs [7]. In mammalian cells, autophagy plays dual roles.

The PI3K/Akt/mTor pathway is important for autophagy; the pathway has become a new

\footnotetext{
${ }^{1}$ This research was supported by Jilin province education department science and technology research projects (2014-192). The authors declare that they have no competing interests in this research.

* Address for correspondence: Yali Qi, Department of Epidemiology, School of Public Health, Beihua University, Jilin 132011, Jilin, China. Tel.: 0432-64608337; Fax: 0432-64608116; E-mail: 374494617@ qq.com.
} 
therapeutic option in some diseases. Akt has serine-threonine kinase activity, which mostly locating at cytoplasm in resting cells. Activation of PI3K may transfer signaling to mTOR by Akt, and then regulate downstream protein activity. Constitutive activation of the PI3K/Akt/mTOR axis is a prototypic survival mechanism commonly encountered in cancer [8]. Overexpression of Akt, constitutive activation of tyrosine kinase growth factor receptors, and exposure to carcinogens, can result in abnormal activation of this pathway, resulting in autophagy suppression [9].

\section{Material and methods}

\subsection{MCF-7, Akt-MCF-7 and Akt RNAi-MCF-7 culture and reagents}

We cultured the MCF-7 cell line (Institute of Biochemistry and Cell Biology, SIBS, CAS), Akt-MCF-7 cell line (Akt overexpressed, by Ph.D. Liu Xiaodong), and Akt-RNAi-MCF-7 cell line (Akt knocked, by Ph.D. Liu Xiaodong) in Dulbecco's Modified Eagle Medium (Gibco, USA) at $37^{\circ} \mathrm{C}$. Antibodies included MAP LC3 (\#12513, Cell signaling, USA), GAPDH (\#sc-365062, Santa Cruz, USA) and Akt (\#sc-5298, Santa Cruz, USA). In addition, peroxidase-conjugated IgG (Santa Cruz, USA), Monodansylcadaverine (MDC) and 3-Methyladenine (3-MA) (Sigma, USA), Z-VCD-FMK (Promega, USA), Annexin V-FITC kit (BD Biosciences, USA) were used.

\subsection{Irradiation}

An X-ray generator (Model XSZ-Z20/20, Dandong, Liaoning, China) delivered radiation at a dose rate of $0.342 \mathrm{~Gy} / \mathrm{min}(200 \mathrm{kV} ; 18 \mathrm{~mA})$.

\subsection{Flow cytometry}

MCF-7 Cells were seeded at $2 \times 10^{4}$ cells per well across 24 well plates, and treated with 3 -MA (2 $\mathrm{mmol} / \mathrm{L})$ and Z-VCD-FMK $(1 \mathrm{mmol} / \mathrm{L})$ for $45 \mathrm{~min}$ at $37^{\circ} \mathrm{C}$; Akt-MCF-7 and Akt-RNAi-MCF-7 achieved $80 \%-90 \%$ fusion. Cells were irradiated by 4 Gy. After $24 \mathrm{~h}$, cells were treated following protocol. After blending, they were incubated for $30 \mathrm{~min}$ in the dark at $37^{\circ} \mathrm{C}$. The stained cells were determined by flow cytometry (Becton, Dickinson and Co., USA).

\subsection{MDC staining assay}

Cells were seeded on coverslips overnight, then cells were treated by 3-MA and Z-VCD-FMK, at 12 or $24 \mathrm{~h}$ after $4 \mathrm{~Gy}$ irradiation. Autophagic vacuoles were labeled with $\mathrm{MDC}(50 \mathrm{mmol} / \mathrm{L})$ at $37^{\circ} \mathrm{C}$ for 1 h. Cells then were washed with PBS and fixed with a solution of $4 \%$ paraformaldehyde for $15 \mathrm{~min}$. The solution was then discarded, and slips were mounted and dried, and observed using fluorescence microscopy (Olympus XSZD2, JAPAN). If each cell had more than 8 autophagic points, they were recognized as achieving autophagy. 150 cells were observed.

\subsection{Western blotting}

After the total protein was extracted with a RIPA lysis buffer, $30 \mu \mathrm{g}$ of protein were separated by SDS-PAGE and transferred to a nitrocellulose membrane (Bio-Rad, USA), which was blocked in PBS 
containing $5 \%$ non-fat dry milk. The membrane was incubated at $4{ }^{\circ} \mathrm{C}$ with antibodies overnight, then washed two times with Tris-buffered saline containing 0.05\% Tween 20 (TBST, pH 7.2) and incubated with horseradish peroxidase-conjugated at $37^{\circ} \mathrm{C}$ for $1 \mathrm{~h}$. The antigen-antibody complex was visualized with ECL system (Santa Cruz, USA).

\subsection{Quantitative real-time PCR detection}

The total RNA was extracted with a TRIzol reagent (Invitrogen, USA). Then, the complementary DNA (cDNA) was synthesized. The following primers were used:

- Glyceraldehydes-3-phosphate dehydrogenase (GAPDH)

- Forward primer 5'-GCACCGTCAAGGCTGAGAAC-3'

- Reverse primer 5'-TGGTGAAGACGCCAGTGGA-3'

- Akt forward primer 5'-CTGAGATTGTGTCAGCCCTGGA-3'

- Reverse primer 5'-CACAGCCCGAAGTCTGTGATCTTA-3'

The PCR protocol is as follows: $95^{\circ} \mathrm{C}$ for $30 \mathrm{~s}, 1$ cycle; $95^{\circ} \mathrm{C}$ for $5 \mathrm{~s}, 60^{\circ} \mathrm{C}$ for $20 \mathrm{~s}, 40$ cycles.

\subsection{Statistical analysis}

Statistical evaluations are presented as mean \pm SD. Data was analyzed using the Student's t-test for statistical significance using SPSS v17.0 software. P values were considered significant if $P<0.05$.

\section{Results}

\subsection{Effects of autophagy/apoptosis inhibitor on IR-induced responsiveness in MCF-7 cells}

As shown in Figure 1A, MAP LC3 II protein increased in 4 the Gy IR group, decreased in the 3-MA group, and had no obvious change in this z-VAD-FMK group. These results indicate that IR-induced apoptosis inhibition cannot increase autophagy. Further combination with the 4 Gy IR caused the MAP LC3 II protein to increase, indicating that IR can increase autophagy in MCF-7 cells where apoptosis was inhibited. Under the same experimental conditions, IR can induce apoptosis. When autophagy was inhibited, MCF-7 cell apoptosis also increased (Figure 1B).

\subsection{Effects of autophagy/apoptosis inhibitor combined with 4 Gy IR on Akt expression in MCF-7 cells}

As shown in Figure 2A, 4 Gy IR-induced Akt mRNA decreases in MCF-7 cells $(P<0.001)$, and combination with autophagic inhibitor 3-MA further deceased Akt mRNA. However, combination with apoptotic inhibitor z-VAD-FMK increased Akt mRNA $(P<0.001)$. In addition, Akt protein decreased after 4 Gy IR, but combination with 3-MA and z-VAD-FMK increased Akt proteins (Figure 2B). 

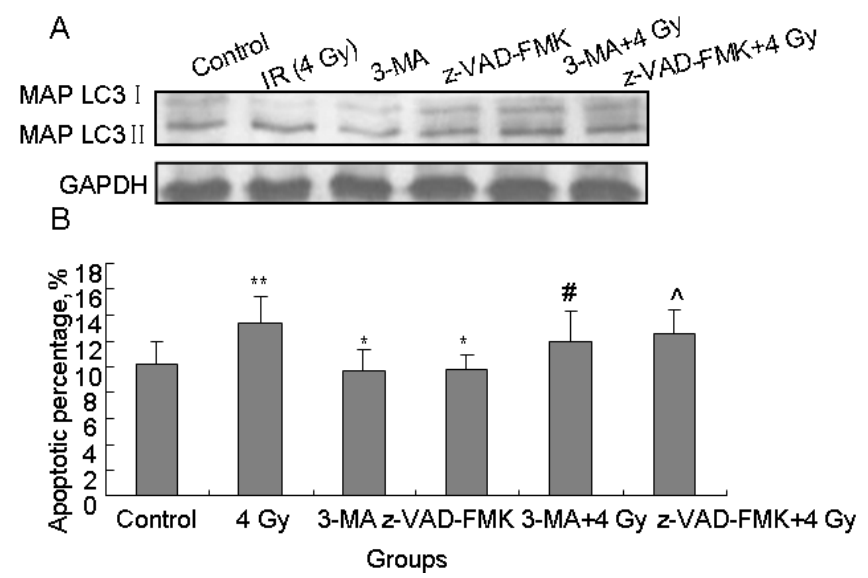

Fig. 1. The relationship between autophagy and apoptosis. (A) Western blotting analysis of MAP LC3 in MCF-7 cells after treatment of -3MA, z-VAD-FMK and 4 Gy IR. (B) Flow cytometry was used to quantify the apoptotic rate after treatment of -3MA, z-VAD-FMK and 4 Gy IR. Data showed mean $\pm \mathrm{SD},{ }^{*} P<0.05$ versus the control, ${ }^{\#} P<0.05$ versus the 3-MA group, and $\wedge P<0.05$ versus the $z-V A D-F M K$ group.

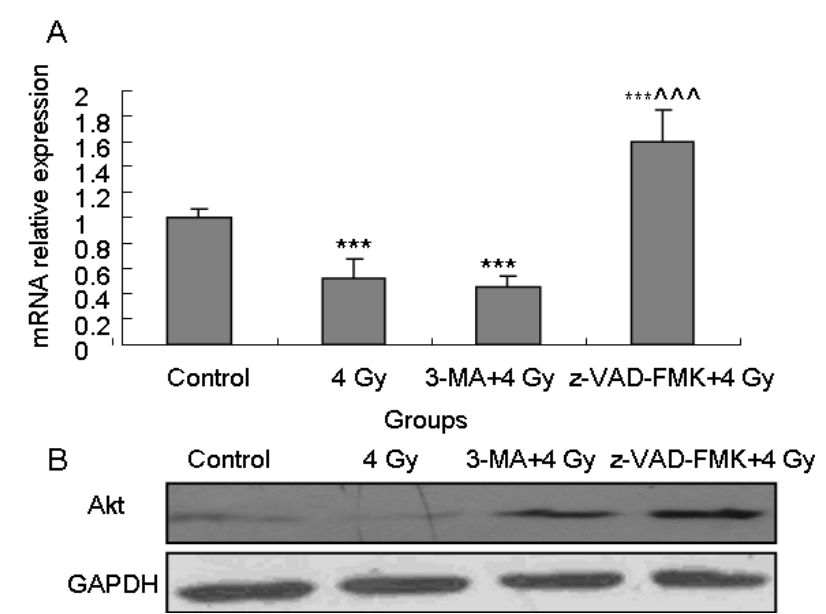

Fig. 2. Change of Akt mRNA and protein. (A) Akt mRNA at different groups by quantitative real-time PCR. (B) Akt protein at different groups by Western blotting. Data showed mean $\pm \mathrm{SD}, * * * P<0.001$ versus the control, \#\#\#P $<0.001$ versus the 3-MA group.

\subsection{Effects of Akt overexpression and being knocked out on autophagy and apoptosis induced by IR}

As shown in Figures $3 \mathrm{~A}$ and 3B, Akt protein increased MCF-7-Akt cells, decreased MCF-7-Akt-RNAi cells, and had no obvious changes on MCF-7-RNAi-empty vector cells. When MCF-7, MCF-7-Akt, and MCF-7-Akt-RNAi were irradiated by 4 Gy. Compared with the control group, apoptotic percentage significantly increased $(\mathrm{P}<0.05, \mathrm{P}<0.001)$; compared with $4 \mathrm{~Gy}$, it significantly decreased in MCF-7-Akt and increased in MCF-7-Akt-RNAi $(\mathrm{P}<0.05)$. This indicated that Akt down-regulated IR-induced apoptosis (Figures 3C and 3D). In addition, compared with the control group, autophagy significantly increased in 4 Gy and the MCF-7-Akt-RNAi group $(\mathrm{P}<0.05$, $\mathrm{P}<0.001$ ). Compared with $4 \mathrm{~Gy}$, it significantly decreased in the MCF-7-Akt group but significantly 
increased in the MCF-7-Akt-RNAi group $(\mathrm{P}<0.05)$. This indicated that Akt down-regulated IR-induced autophagy (Figures $3 \mathrm{E}$ and $3 \mathrm{~F}$ ).

A

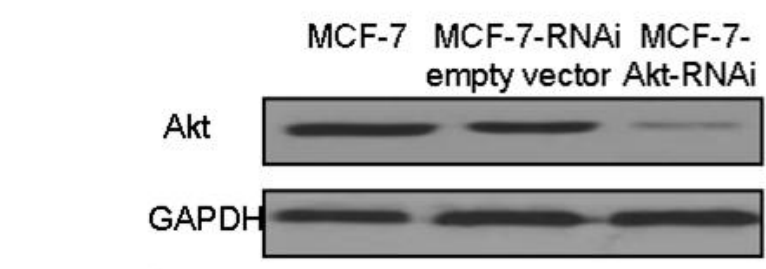

C

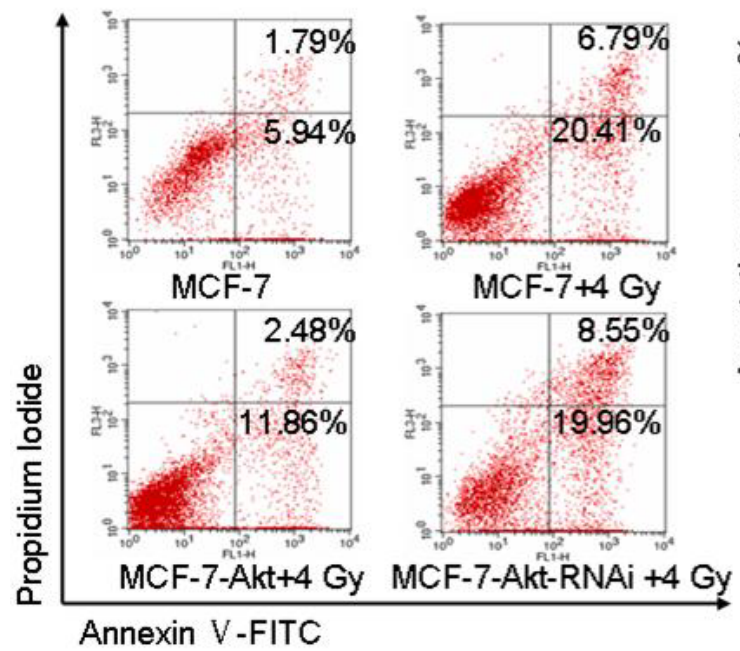

E

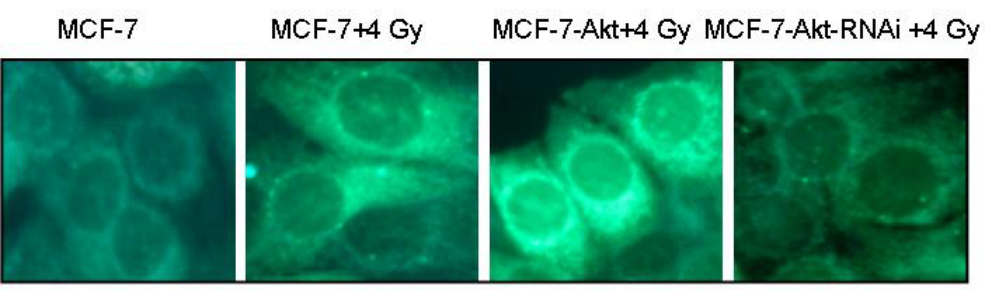

\section{MCF-7 MCF-7-Akt}

Akt

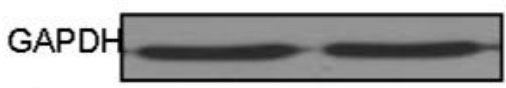

D

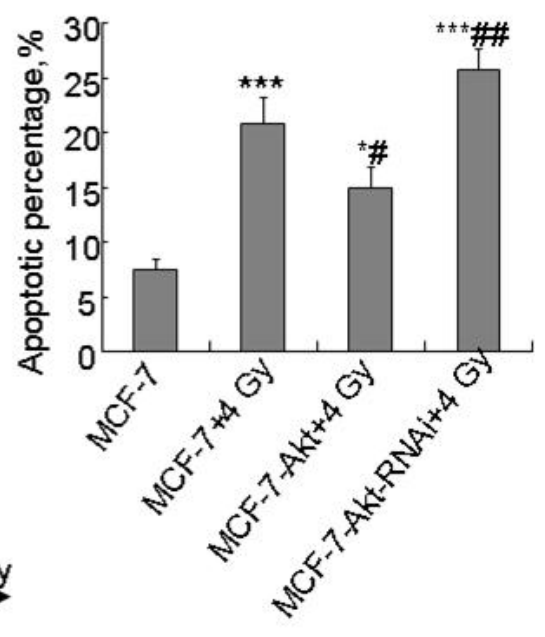

$\mathrm{F}$

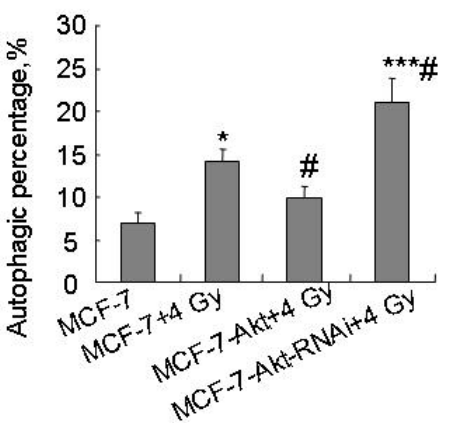

Fig. 3. Effects of Akt on autophagy and IR-induced apoptosis. (A) and (B) Akt proteins in the MCF-7-Akt and MCF-7-Akt-RNAi cell models by Western blotting, respectively. (C) Flow cytometry was used to quantify the apoptotic percentage. Cells were stained with PI and Annexin V-FITC. The positive-stained cells were counted using FACScan. (D) Quantitative analysis of apoptotic percentage. (E) Representative images of MDC positive MCF-7 cells, $\times 200$. (F) Quantitative analysis of autophagy. Data showed mean $\pm \mathrm{SD}, * \mathrm{P}<0.05, * * * \mathrm{P}<0.001$ versus control group, \#P $<0.05$, and $\# \# \mathrm{P}<0.001$ compared with $4 \mathrm{~Gy}$. 


\section{Discussion}

Radiotherapy is an important means of breast cancer therapy. Radiation kills cancer cells mainly by inducing necrosis and apoptosis. Successfully inhibiting the growth of cancer cells using radiothereutic strategies is mostly dependent on the ability to trigger tumor cell apoptosis $[10,11]$. However, some studies have found that the apoptosis induced by ionizing radiation is reduced in cancer cell lines lacking apoptosis gene caspase-3 and -9; instead, radiation-inducing autophagy is promoted. In our study, we confirmed that IR could induce autophagy in the MCF-7 cell, but IR could also induce cell apoptosis. In addition, autophagic inhibitor 3-MA and apoptotic inhibitor z-VAD-FMK was used on MCF-7 cells. We found that IR can induce autophagy and apoptosis in MCF-7 cells; apoptosis inhibition cannot increases autophagy, but inhibition of autophagy can increase apoptosis. This might relate to the pro-survival role of autophagy in cancer cells. It has been suggested that inhibiting autophagy can enhance the radiation-induced death role on MCF-7 cells and provide a new strategy for cancer radiotherapy.

As an alternative cell death pathway to apoptosis, autophagy is currently an important research target for therapy. The rapid advancement in understanding the mechanisms and regulation of autophagy has placed this process at the center of current research. Unfortunately, the expression of caspase-3 was about $75 \%$ of breast cancer tissues down-regulates or lacks. Thus, caspase-3-deficient breast cancer cells, such as MCF-7, were resistant to radiotherapy [12]. Autophagy signaling pathways referred to PI3K-I/Akt/mTOR, PI3K- III / Beclin1 and p53, and so on. Akt is an important downstream target kinase of PI3K; overexpression of Akt can result in abnormal activation of this pathway, resulting in autophagy suppression. In addition, the activation of PI3K/Akt/mTOR can also inhibit cell apoptosis; it may promote radio- and chemo-therapy roles by inhibiting the activity of this pathway. Therefore, targeting Akt to improve the effects of radiation on MCF-7 cells has great significance in clinical radiotherapy. After 4 Gy IR, Akt mRNA and protein decreased in MCF-7 cells, inhibition autophagy and apoptosis combined with 4 Gy could induce an increase in Akt protein, but only apoptosis inhibition combined with 4 Gy could induce an Akt mRNA increase. This indicated that Akt has a down-regulating role on autophagy and apoptosis. There are some disputes about the role of radiation-inducing autophagy on cancer; some studies demonstrate that the inhibition of autophagy could improve cancer radiosensitivity [13]. Further, we found that the overexpression of Akt reduced radiation-induced autophagy and apoptosis, but Akt down-regulation had the opposite effects on autophagy and apoptosis.

In summary, we observed that radiation could induce autophagy and apoptosis in MCF-7 cells, and inhibition of autophagy could increase apoptosis. Moreover, we found Akt had a down-regulation role on autophagy and apoptosis. After Akt was removed, radiation-induced autophagy and apoptosis increased. Our findings provide new possibilities for breast cancer radiotherapy.

\section{Acknowledgments}

We thank for Professor LIU Xiaodong who come from the key laboratory of radiobiology, ministry of health, school of public health, Jilin University. This study was supported by the National Natural Science Foundation of China (30970681), Young Scholars Program of Norman Bethune Health 
Science Center of Jilin University (2013202017), and Jilin Province Education Department of Science and Technology Research Projects (2014-192) .

\section{References}

[1] C. DeSantis, R. Siegel, P. Bandi, et al., Breast cancer statistics, .CA: A Cancer Journal for Clinicians 61 (2011), 409-418.

[2] A. Apel, I. Herr, H. Schwarz, et al., Blocked autophagy sensitizes resistant carcinoma cells to radiation therapy, Cancer Research 68 (2008), 1485-1494.

[3] P. Giménez-Bonafé, A. Tortosa, R. Pérez-Tomás, et al., Overcoming drug resistance by enhancing apoptosis of tumor cells, Current Cancer Drug Targets 9 (2009), 320-340.

[4] E.C. Ledgerwood and I.M. Morison, Targeting the apoptosome for cancer therapy, Clinical Cancer Research 15 (2009), 420-424.

[5] X.H. Yang, S. Edgerton and A.D. Thor, Reconstitution of caspase-3 sensitizes MCF-7 breast cancer cells to radiation therapy, International Journal of Oncology 26 (2005), 1675-1680.

[6] D.L. Kwong, J.S. Sham, L.H. Leung, et al., Preliminary results of radiation dose escalation for locally advanced nasopharyngeal carcinoma, International Journal of Radiation Oncology, Biology, Physics 64 (2006), 374-381.

[7] K.W. Kim, L. Moretti, L.R. Mitchell, et al., Endoplasmic reticulum stress mediates radiation-induced autophagy by perk-eIF2alpha in caspase-3/7-deficient cells, Oncogene 29 (2010), 3241-3251.

[8] P. Giménez-Bonafé, A. Tortosa, R. Pérez-Tomás, et al., Overcoming drug resistance by enhancing apoptosis of tumor cells, Current Cancer Drug Targets 9 (2009), 320-340.

[9] R. Sahu, S. Kaushik, C.C. Clement, et al., Microautophagy of cytosolic proteins by late endosomes, Developmental Cell 20 (2011), 131-139.

[10] W.K. Wu, S.B. Coffelt, C.H. Cho, et al., The autophagic paradox in cancer therapy, Oncogene 31 (2012), 939-953.

[11] T. Kirkegaard, C.J. Witton, J. Edwards, et al., Molecular alterations in AKT1, AKT2 and AKT3 detected in breast and prostatic cancer by FISH, Histopathology 56 (2010), 203-211.

[12] W. Li, Q. Yang and Z. Mao., Chaperone-mediated autophagy: Machinery, regulation and biological consequences, Cellular and Molecular Life Sciences 68 (2011), 749-763.

[13] C.S. Lin, Y.C. Wang, J.L. Huang, et al., Autophagy and reactive oxygen species modulate cytotoxicity induced by suppression of ATM kinase activity inhead and neck cancer cells, Oral Oncology 48 (2012), 1152-1158. 